\title{
Dual-frequency division de-multiplexer based on cascaded photonic crystal waveguides
}

\author{
Ahmet E. Akosman ${ }^{\mathrm{a}, *}$, Mehmet Mutlu ${ }^{\mathrm{a}}$, Hamza Kurt ${ }^{\mathrm{b}}$, Ekmel Ozbay ${ }^{\mathrm{a}}$ \\ ${ }^{a}$ Department of Electrical and Electronics Engineering, Nanotechnology Research Center, Bilkent University, 06800 Ankara, Turkey \\ ${ }^{\mathrm{b}}$ Department of Electrical and Electronics Engineering, TOBB University of Economics and Technology, 06560 Ankara, Turkey
}

\section{A R T I C L E I N F O}

Available online 28 February 2012

Keywords:

Photonic crystal

Slow light

Optical communication

De-multiplexer

\begin{abstract}
A B S T R A C T
A dual-frequency division de-multiplexing mechanism is demonstrated using cascaded photonic crystal waveguides with unequal waveguide widths. The de-multiplexing mechanism is based on the frequency shift of the waveguide bands for the unequal widths of the photonic crystal waveguides. The modulation in the waveguide bands is used for providing frequency selectivity to the system. The slow light regime of the waveguide bands is utilized for extracting the desired frequency bands from a wider photonic crystal waveguide that has a relatively larger group velocity than the main waveguide for the de-multiplexed frequencies. In other words, the wider spatial distribution of the electric fields in the transverse direction of the waveguide for slow light modes is utilized in order to achieve the dropping of the modes to the output channels. The spectral and spatial de-multiplexing features are numerically verified. It can be stated that the presented mechanism can be used to de-multiplex more than two frequency intervals by cascading new photonic crystal waveguides with properly selected widths.
\end{abstract}

(c) 2012 Elsevier B.V. All rights reserved.

\section{Introduction}

The wavelength sensitivity of the photonic crystals (PCs) has attracted numerous researchers to utilize such structures in optical communication systems [1-4]. One of the main components of the optical communication systems is the wavelength division de-multiplexing (WDM) devices. De-multiplexers (DEMUXs) are devices that separate a given input source either spatially or spectrally to the output channels. Contemporary studies on WDM devices are targeted to achieve ultra compact, polarization insensitive devices with a low cross-talk ratio between the output channels [5,6]. Among the efforts in the literature that aim to create a de-multiplexing mechanism based on PC structures, PC waveguide directional couplers for de-multiplexing the desired wavelengths in the coupled parallel waveguides with different perturbations to create different resonance frequencies $[7,8]$, PC based frequency selective micro cavities with different hole sizes to target different resonance frequencies [9], coarse wavelength de-multiplexers that have multimode waveguides in order to create a mini stop band from the interaction of the main waveguide mode with the higher order mode and to provide a difference in the spatial distributions of

\footnotetext{
* Corresponding author. Tel.: +90 312 2901019; fax: +90 3122901015.

E-mail address: akosman@ee.bilkent.edu.tr (A.E. Akosman).
}

the modes that have different group velocities [10,11], classical bulk PCs by the usage of the self-imaging property [12], and classical bulk PCs that utilize the super-prism phenomenon for benefiting from the negative refraction to direct a set of targeted frequencies in the desired directions and thus creating a spatial and spectral de-multiplexing effect $[13,14]$ should be mentioned. The method of cascading PC sections with unequal periodicities and waveguide widths is also used to achieve the extraction of different frequency bands from different output channels utilizing coupled cavity-waveguide combinations $[15,16]$. Furthermore, a recent study proposes a general framework for optical de-multiplexing in two dimensional photonic crystals benefiting from waveguide-waveguide coupling [17].

In a previous study, we demonstrated the implementation of a different approach that benefits from the spatial distribution characteristics of the waveguide modes for the purpose of obtaining the desired frequencies from the corresponding cascaded photonic crystal waveguide (PCW) blocks [18]. In the present article, we study the potential of combining the ultra-compact single frequency de-multiplexing operation proposed in ref. [18] and the modulation of the waveguide bands by modifying the waveguide widths, which is proposed in Refs. [11,16]. It should be noted that the dielectric constants and the periodicity of the consecutive $\mathrm{PC}$ sections are not varied for the purpose of achieving frequency division de-multiplexing. In addition, the possibility of obtaining a better low cross-talk ratio compared to 
the study given in Ref. [18] is investigated and this aim is achieved by adjusting the cut-off frequencies of each waveguide band with the careful optimization of the parameters.

\section{The models and the method}

The de-multiplexing mechanism in this study is designed and optimized for achieving frequency division operation in a specific frequency interval by the involvement of the slow light regime of cascaded PCWs. The main purpose in this study is to create an ultra-compact design with a very small footprint that can be achieved by the utilization of PC based structures. Moreover, the entire de-multiplexing scheme is bounded such that the frequency bands of interest lie inside the stop band region of the $\mathrm{PC}$ and that the optimization results in a minimized cross-talk ratio between the output channels. Initially, a classical two dimensional PC is designed to create a photonic band gap (PBG) region. Afterwards, one entire row of dielectric rods is removed along the $\Gamma X$ direction from the PC to create a PCW along the propagation direction. As it can be deduced, a modulation of the waveguide bands in the frequency axis is required to achieve frequency selectivity between the cascaded PCWs. We propose that a modulation can be introduced by altering the waveguide widths. Thus, a combination of two PCWs with

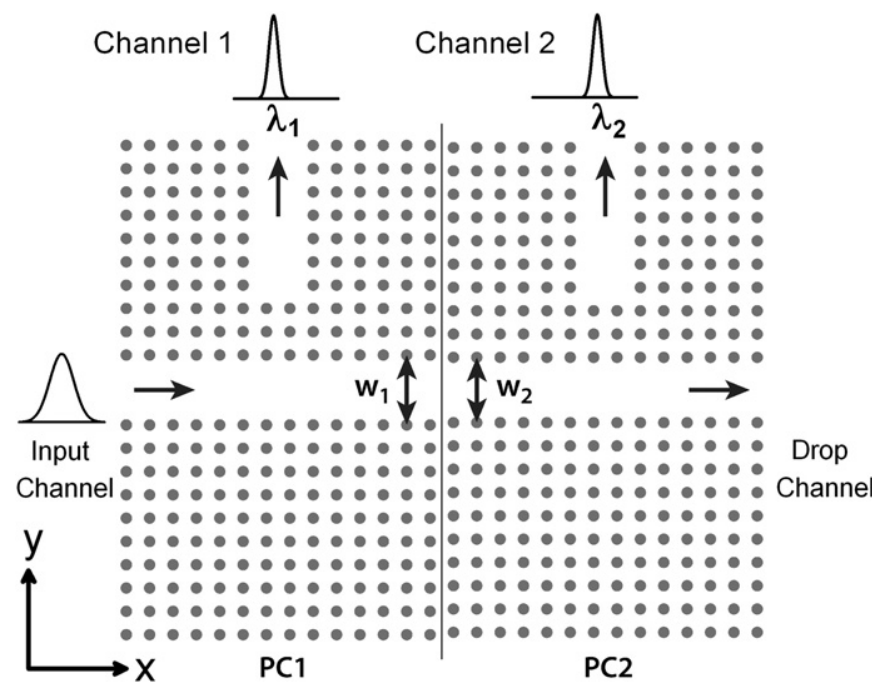

Fig. 1. Geometry of the proposed DEMUX structure. The design consists of two cascaded PCWs with unequal waveguide widths $w_{1}$ and $w_{2}$, respectively. The radii of the dielectric rods and the lattice periodicities are identical. different waveguide widths can be used for suppressing specific frequencies that are not desired in the next PCW. After the construction of a frequency selective cascaded waveguide scheme, the introduction of output channels is necessary for the extraction of the de-multiplexed frequencies. At this point, we implement a novel mechanism for the extraction of the desired frequencies that includes the utilization of the spatial distribution characteristics of the slow light modes inside the PCW that correspond to the close vicinity of the $k=0$ band edge of the Brilliouin zone of the corresponding PCW. The field distributions of the slow light modes along the direction that is perpendicular to the propagation direction are spatially more broadened as a consequence of the increase in the oscillations in that direction. It is intuitive to predict that opening a new waveguide along the perpendicular direction gives rise to the leakage of the modes that have broadened field distribution along the transverse plane by evanescent coupling to the new waveguide. Therefore, the output channels are formed by structuring a new PCW that is created by removing extra rods along the perpendicular direction. The output channels are designed to have a waveguide width of $3 a$, where $a$ is the periodicity of the PC structure, that corresponds to the removal of two entire rows of dielectric rods. Although the output waveguide exhibits multi-mode propagation, it is necessary that it has a width that is larger than the main waveguide width in order to realize a gradual impedance transition for the de-multiplexed frequencies that results in an optimal coupling between the waveguides. To sum up, the proposed design is finalized after forming the output channels that provide for the extraction of the desired frequencies.

The geometry of the proposed DEMUX design is depicted in Fig. 1. Two PCWs that contain rods of the same radii and have the same lattice periodicity but unequal waveguide widths are cascaded. The unequal waveguide widths generate a modulation in the waveguide bands that makes each PCW sensitive to a different band of frequencies and different regions of the slow light modes. The frequency sensitivity also comes from the nature of the PC structure that prevents the propagation of the modes that lie inside the PBG region.

The proposed structure can be investigated by starting with a classical two dimensional PC structure. The periodicity of the lattices and the dielectric constant of the dielectric rods are selected as $r / a=0.24$ and $\varepsilon=12$, respectively. The dispersion diagram of the PC structure with the given lattice periodicity and the dielectric constant value is shown in Fig. 2(a). The dispersion characteristics of the PC are determined using the Plane-Wave Expansion Method (PWM). The structure has a PBG between the normalized frequencies of 0.25 and 0.38 . The removal of an entire row of rods in the $x$-direction results in a a

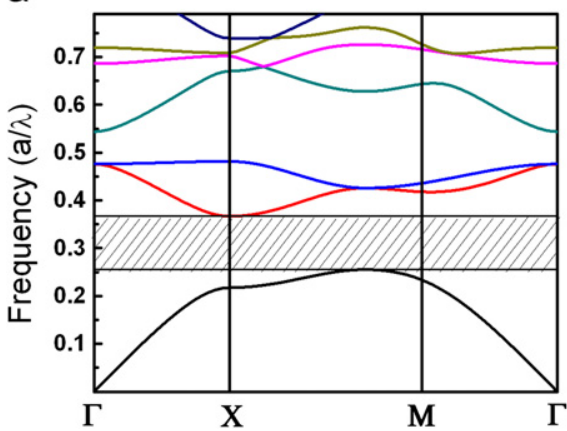

b

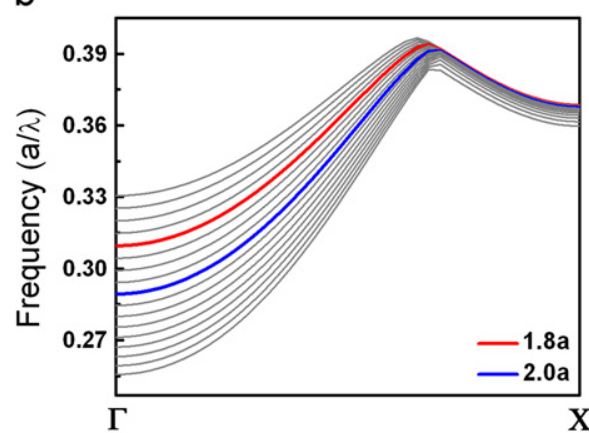

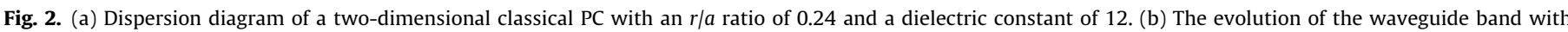

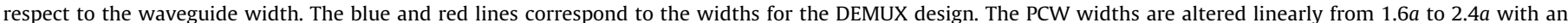
increment of $0.05 a$. (For interpretation of the references to color in this figure legend, the reader is referred to the web version of this article.) 
set of allowed modes in the vicinity of the normalized frequencies of 0.285 and 0.39 that form a waveguide band. The cut-off frequency of the waveguide band can be modulated by the alteration of the waveguide width. In the numerical calculations, the width of the waveguide is changed between $1.6 a$ and $2.4 a$. The PBG region remains approximately constant since the total filling factor of the PC structure does not change while altering the channel width. However, the position of the waveguide band on the frequency axis changes as a result of the modification of the waveguide width. The evolution of the waveguide band with respect to the waveguide width is shown in Fig. 2(b). For all the waveguide widths, the cut-off frequencies of the waveguide bands lie inside the PBG region and that provides a large parametric interval for optimizing the design such that it exhibits the desired DEMUX behavior. The expected de-multiplexed frequency intervals are the slow light modes on the waveguide band that correspond to the flat regions of the waveguide band. Therefore, the widths of the cascaded PCWs should be selected to be separated enough such that the slow light regions do not coincide. Accordingly, the waveguide widths of the PCWs are chosen as $w_{1}=2 a$ and $w_{2}=1.8 a$. This cascaded waveguide structure provides two separated slow light regions for each one of the cascaded PCW sections. Finally, an extraction mechanism is required in order to direct these slow light modes into different output channels.

A multimode PCW is designed as an output channel for each PCW section. Indeed, a single mode classical PCW can serve the same mechanism to the system, but the underlying reason for this choice can be explained by inspecting the dispersion characteristics of the designed output channel. Firstly, the output channels are constructed by removing two rods along the $y$-direction to drop the slow light modes using their broadened spatial distribution along the $y$-direction. Secondly, it is known that the group velocities of the targeted frequency interval are lower than the other modes that are allowed to propagate in the PCW. Thus, the impedance mismatch between the air and the structure for the targeted modes is larger than the other allowed frequencies that in turn result in a significant amount of reflection in the coupling process between the main PCW and the output channels. A gradual transition in the impedances is needed to couple the targeted slow light modes into the free space efficiently. Moreover, it is known that a broader PCW exhibits a set of allowed modes that have higher group velocities than a classical PCW with a waveguide width of $w=2 a$. Hence, such a broad PCW can be a good candidate for a more optimized power extraction through the output channel. Finally, a PCW is designed with a waveguide width of $3 a$ in order to propose a simpler design and to create a gradual impedance transition for the slow light modes between the main channel and the air. The DEMUX design is finalized by the formation of the output channels.

\section{Numerical examples and discussions}

The transmission spectra of the output channels are given in Fig. 3. The numerical analysis of the proposed design is performed using FDTD SOLUTIONS (version 6.0) from Lumerical Solutions, Inc. (Vancouver, Canada), which is a commercial software program based on the finite difference time domain method (FDTD). The geometrical parameters and the dielectric constant of the design are given in the previous section. The input source is chosen as a Gaussian wave that has a wide range of frequency components in order to cover the entire PBG region of the classical PC. The input source is positioned far enough from the DEMUX structure in order to illuminate the structure with almost flat phase fronts for achieving an approximate plane wave

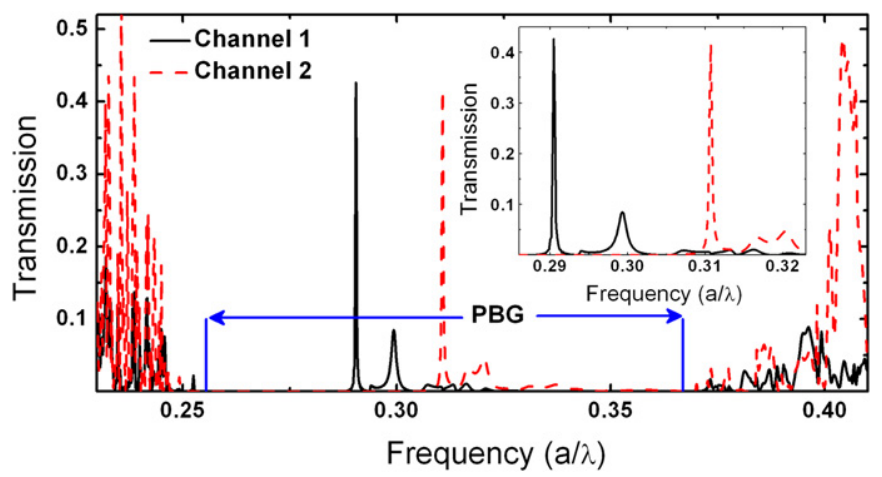

Fig. 3. Transmission spectra of the output channels. The inset shows the enlarged view of the de-multiplexed frequency intervals.

illumination. The electric field intensity is monitored at the output of each output channel. The intensity values are normalized with respect to the intensity of the input source. Fig. 3 clearly shows that the extraction of the slow light modes inside each PCW section can be achieved with a very high quality factor.

The transmission through the output channels is restricted by the PBG region and the slow light region as explained in detail hereinabove. However, there is a second transmission peak with a much lower intensity for each output channel that corresponds to faster modes compared to the narrow transmission peaks. The occurrence of the second peak is related to the back reflections at the output of the each PCW section. This transmission which is due to the internal reflections inside the main channel can be decreased by creating longer PCW sections that can be the subject of further optimization. It is observed that the extraction of modes other than the slow light modes in channel 2 is much less than channel 1 . This observation shows that the reflection in the second PCW section is less crucial than the reflections from the interface between the PCW sections. Moreover, the de-multiplexed frequency intervals at each output channel do not overlap and the center frequencies are similar to the cut-off frequencies of the waveguide bands shown in Fig. 2(b) and this shows that the results derived from the PWM and FDTD methods are consistent. One can see that the center frequencies of the extracted slow light modes are not exactly the same as the cut-off frequencies of the corresponding waveguide widths but are slightly higher. The reason behind that can be explained by the nature of the modes at the $k=0$ band edge. The modes at the $k=0$ band edge have very large group indices that correspond to a high effective dielectric constant that can result in an approximately unity reflection at the PCW input. Therefore, the peak of the transmission at the output channel slightly shifts to higher frequencies. The transmission is also restricted by the waveguide modes with higher frequencies because their group indices decrease with the increase in the frequency and their field distributions in the transverse direction become narrower and which gives rise to a decreased coupling between the main channel and the output channels. Furthermore, these two limitations on the de-multiplexed frequency intervals result in a narrowband transmission in frequency. The quality factors of the de-multiplexed frequency intervals are calculated approximately as 1930 and 1550 for channels 1 and 2, respectively.

The spatial distributions of the de-multiplexed frequencies are shown in Fig. 4. The behavior of the slow light modes in the main channel and the coupling between the main channel and the output channel can be clearly observed. The investigated frequencies are selected such that they are the frequencies of peak transmission of the de-multiplexed frequency bands for each output channel. The separation between the cut-off frequencies 

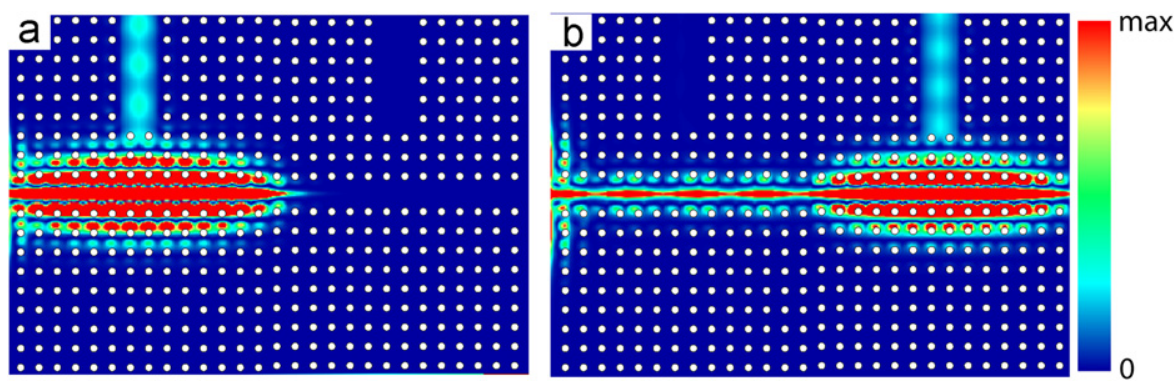

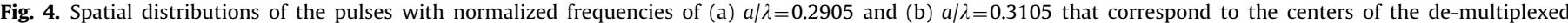
frequency bands at PC1 and PC2 sections, respectively.

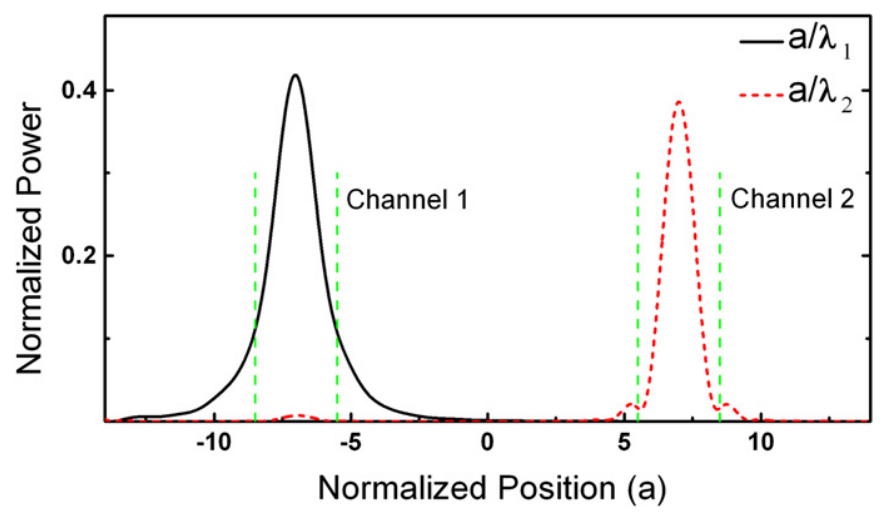

Fig. 5. Spatial distributions of the center frequencies of the de-multiplexed frequency bands at the output channels along the $x$-direction. The vertical green dashed lines correspond to the positions of the output channels. (For interpretation of the references to color in this figure legend, the reader is referred to the web version of this article.)

of the waveguide modes of the chosen PCW sections can be seen in Fig. 4(a) as zero transmission through the boundary of PC1 and PC2. Moreover, the broad spatial distributions of the slow light modes for both channels are clearly demonstrated. In Fig. 4(b), the propagation of the frequencies to be de-multiplexed in channel 2 along the cascaded structure is shown. It is an allowed waveguide mode with a moderate group index whereas it becomes a slow light mode after entering the waveguide section of PC2.

We also examined the spatial distributions of the center frequencies of the de-multiplexed frequency bands along the $x$-direction at the output of the output channels and these results are shown in Fig. 5. The degree of the cross-talk between output channels can be calculated by the usage of the electric field intensities along the $x$-direction. The maximum cross-talk values for channels 1 and 2 are calculated as $-20 \mathrm{~dB}$ and $-40 \mathrm{~dB}$, respectively. The restrictions on the de-multiplexed frequencies result in a very low cross-talk between the channels. However, the underlying reason for having obtained such small values for the cross-talk is the modulation of the waveguide bands that is performed by changing the waveguide widths. The slight difference in the cut-off frequencies causes the de-multiplexed frequencies to be prohibited for the next PCW section. In the proposed design, the de-multiplexed frequencies in channel 1 are already in the stop band of channel 2 which yields a very low cross talk for channel 1 . The reason for the low cross-talk for channel 2 is the physical mechanism of the de-multiplexing operation. The de-multiplexed frequencies in channel 2 propagate with moderate group indices along the waveguide of the PC1 section. Therefore, they cannot be dropped along channel 1 as a consequence of their narrow spatial distribution along the $y$-direction.
The de-multiplexing operation is investigated and achieved utilizing the slow light regime of PCWs. Further optimizations can be performed in order to create a design with narrower de-multiplexed frequency bands, a larger extracted power and a lower cross-talk ratio between the channels. The length of the PCWs can be modified in order to obtain different center frequencies. The waveguide widths of the cascaded PCW sections can be adjusted in order to modulate the de-multiplexed frequencies inside the PBG region. The number of the PCW sections can be increased for the purpose of de-multiplexing more than two sets of frequency bands. The back reflections inside the waveguides can be minimized by a further careful optimization of the geometrical properties of the PCWs.

In this study, the utilization of a high permittivity dielectric has two significant advantages. Firstly, introducing a higher dielectric contrast between the periodic structure and the background medium results in a larger PBG and enables the easier control of modulation by the modification of the PC waveguide width. Secondly, the waveguide bands have smaller slopes at the $k=0$ band-edge due to the usage of high permittivity dielectrics and that result in slower modes. However, a similar mechanism can be obtained using materials with smaller dielectric constants, which are more appropriate concerning the optical frequencies, with some drawbacks such as a narrower PBG region, which corresponds to a less flexible design, and de-multiplexed frequency intervals with lower quality factors as an implication of the lower group indices at the operating frequencies.

\section{Conclusions}

A frequency division de-multiplexer, which benefits from the slow light regime of the photonic crystal waveguides and is formed by cascading waveguides of unequal widths, is proposed in this study. The de-multiplexing mechanism originates from the spatial distribution characteristics of the slow light modes along the photonic crystal waveguides. The wavelength selectivity of the cascaded photonic crystal sections is obtained by changing the waveguide width of each section. This waveguide band modulation also yields the spatial selectivity of the de-multiplexed frequency bands. The spatial and spectral properties of the de-multiplexed frequency bands are numerically investigated and demonstrated. One strict limitation for the usage of slow light modes is the relatively low extracted power from output channels as a consequence of the high effective dielectric permittivity encountered by the slow light modes compared to the previous de-multiplexing efforts. In spite of the fact that the proposed design is optimized to achieve maximum coupling by introducing a gradual transition in the effective dielectric permittivities between the main channel and the output channels, input and output couplers can be appended to the current geometry for the purpose of smoothing the transition of effective permittivities 
between the air and the PC design for enabling the obtaining of increased extracted power. The utilization of the slow light regime for de-multiplexing purposes has several advantages at first glance. Firstly, the de-multiplexers that are based on PC structures generally require the occupation of large areas for the achievement of spatial selectivity; however, the proposed structure has a very small footprint compared to the other designs. Secondly, the de-multiplexed set of frequencies can have a very narrow bandwidth and therefore, high quality factors can be obtained as a result of the limited slow light regions in the photonic crystal waveguide bands. Finally, a very low cross-talk can be achieved by the utilization of the slow light regime with a proper modulation of the geometries of the consecutive photonic crystal waveguide sections.

\section{Acknowledgments}

This work is supported by the projects DPT-HAMIT, EU-PHOME, EU-N4E, NATO-SET-181 and TUBITAK under Project nos. 107A004, 107A012, and 109E301. H. Kurt acknowledges support from the Turkish Academy of Sciences Distinguished Young Scientist Award (TUBA GEBIP). One of the authors (E.O.) also acknowledges partial support from the Turkish Academy of Sciences.

\section{References}

[1] J.E. Centeno, B. Guizal, D. Felbacq, J. Opt. A—Pure Appl. Opt. 1 (5) (1999) 110.

[2] M. Koshiba, J. Lightwave Technol. 19 (12) (2001) 1970.

[3] A. Sharkawy, S. Shi, D.W. Prather, Appl. Opt. 40 (14) (2001) 2247.

[4] F.V. Laere, T. Stomeo, C. Cambournac, M. Ayre, R. Brenot, H. Benisty G. Roelkens, T.F. Krauss, D. Van Thourhout, R. Baets, J. Lightwave Technol. 27 (2) (2009) 417.

[5] M. Notomi, A. Shinya, S. Mitsugi, E. Kuramochi, H.Y. Ryu, Opt. Express 12 (8) (2004) 1551.

[6] F.V. Laere, T. Stomeo, C. Cambournac, M. Ayre, R. Brenot, H. Benisty, G. Roelkens, T.F. Krauss, D. Van Thourhout, R. Baets, J. Lightwave Technol. 27 (2) (2009) 417.

[7] M. Bayindir, E. Ozbay, Opt. Express 10 (22) (2002) 1279.

[8] S. Boscolo, M. Midrio, C.G. Someda, IEEE J. Quantum Electron. 38 (1) (2002) 47.

[9] S. Fan, P.R. Villeneuve, J.D. Joannopoulos, H.A. Haus, Opt. Express 3 (1) (1998) 4

[10] L. Martinelli, H. Benisty, O. Khayam, G.H. Duan, H. Heidrich, K. Janiak, J. Lightwave Technol. 25 (9) (2007) 2385.

[11] H. Benisty, C. Cambournac, F. Van Laere, D. Van Thourhout, J. Lightwave Technol. 28 (8) (2010) 1201.

[12] H. Kim, I. Park, B.O.S. Park, E. Lee, S. Lee, Opt. Express 12 (23) (2004) 5625.

[13] L. Wu, M. Mazilu, T. Karle, T.F. Krauss, IEEE J. Quantum Electron. 38 (4) (2002) 915.

[14] A. Adibi, R.K. Lee, Y. Xu, A. Yariv, A. Scherer, Electron. Lett. 36 (16) (2000) 1376.

[15] H. Takano, B.-S. Song, T. Asano, S. Noda, Opt. Express 14 (2006) 3491.

[16] A. Shinya, S. Mitsugi, E. Kuramochi, M. Notomi, Opt. Express 14 (2006) 12394.

[17] V. Liu, Y. Jiao, D.A.B. Miller, S. Fan, Opt. Lett. 34 (2011) 591.

[18] A.E. Akosman, M. Mutlu, H. Kurt, E. Ozbay, Opt. Express 19 (2011) 24129. 